\title{
We must overcome the fear of change
}

Is it possible to grow old with good health and quality of life in Brazil? How much does the health system contribute to this? And how can this health system, especially the supplementary system, be redesigned to meet the needs of the population in a short period of time? What needs to be done?

This and other related questions have been the subject of many debates, studies and forums.

The increase in life expectancy in the last decades has been a great achievement for the Brazilian population. Living longer - aging - is already a reality in the current decade, and will become more common in the years to come.

However, the structure of the supplementary health system in Brazil must adjust to the different demographic and epidemiological profiles resulting from the greater participation of the elderly in the population. The scale of the increase in health expenditure on the elderly population will depend, above all, on these additional years being healthy and free from illness and dependence. Prevention, the maintenance of health, independence and autonomy, and delaying disease and frailty in an older population are the major health-related challenges for aging populations. Thus, any social and health policy for the elderly should consider the promotion of health and the maintenance of functional capacity.

Some points must be highlighted in this reorganization of the health system, such as: the training of health professionals (who are still trained and instructed as they were decades ago) focusing on a completely different epidemiological and demographic profile; specialists who are still molded and guided by the prevailing model of remuneration, which prioritizes specialties linked to the execution of procedures, with the role of specialties such as geriatrics and medical and family clinical care diminishing; the lack of health care equipment for elderly care, namely: palliative care, home care, transitional and long-term care, day centers and others; lack of a structured entrance point for the creation of a care plan, avoiding unnecessary emergencies and hospitalizations; the lack of an electronic health record, which stores and makes the entire health/illness history of an elderly person available in all care locations.

All these changes in the health service delivery model need to be accompanied by a change in the way the supplementary health sector is remunerated, moving from a model focused on payment for procedures and services (fee-for-service) to alternatives that make the user the center of health actions (patient-centered).

The reorganization of the care and payment model must generate better care results, as well as the reduction of waste and iatrogenesis, making the system more efficient and ensuring the patient receives better care. 
What is needed to achieve this? We must get out of our comfort zone and away from the "more of the same" approach. We need to innovate in the care we provide, in the way we remunerate the sector and in our evaluation of the quality of the sector. We must always remember that innovating often means recovering the simplest care and values that have been lost within our health system.

Thus, it is not enough to simply argue that the cost of health increases every year, or that aging brings additional costs to the system. It must be understood, finally, that a model that only contributes to inefficiencies must change or costs will become unsustainable. We can no longer put the blame on spending on the elderly or try to expunge the "risk" of health insurance portfolios. There is already strong evidence to show that great results can be achieved by doing things differently. The desire to do so is all that is required.

The proposal behind the "Projeto Idoso Bem Cuidado" ("the Well Cared For Elderly Person Project"), developed by ANS and created in partnership with several institutions, has not only structured this reorganization of health care and compensation models, but has already presented significant improvements in healthcare and economic indicators, demonstrating that sustainability and performance can be achieved.

It's time to bring about the changes required! Everyone will benefit, especially the patient.

Martha Regina de Oliveira

Doctor, Regulatory Specialist and former Director of Sectorial Development of the Agência Nacional de Saúde Suplementar (the National Supplementary Health Agency) (ANS) 